\title{
Intramolecular bonds resolved on a semiconductor surface
}

\author{
Adam Sweetman, ${ }^{1, *}, \dagger$ Samuel P. Jarvis, ${ }^{1, \dagger}$ Philipp Rahe,,${ }^{1,2}$ Neil R. Champness,${ }^{3}$ Lev Kantorovich, ${ }^{4}$ and Philip Moriarty ${ }^{1}$ \\ ${ }^{1}$ The School of Physics and Astronomy, The University of Nottingham, Nottingham, NG7 2RD, United Kingdom \\ ${ }^{2}$ Department of Physics and Astronomy, The University of Utah, Salt Lake City, Utah 84112, USA \\ ${ }^{3}$ The School of Chemistry, The University of Nottingham, Nottingham, NG7 2RD, United Kingdom \\ ${ }^{4}$ Department of Physics, King's College London, The Strand, London, WC2R 2LS, United Kingdom \\ (Received 6 November 2013; revised manuscript received 27 August 2014; published 20 October 2014)
}

\begin{abstract}
Noncontact atomic force microscopy (NC-AFM) is now routinely capable of obtaining submolecular resolution, readily resolving the carbon backbone structure of planar organic molecules adsorbed on metal substrates. Here we show that the same resolution may also be obtained for molecules adsorbed on a reactive semiconducting substrate. Surprisingly, this resolution is routinely obtained without the need for deliberate tip functionalization. Intriguingly, we observe two chemically distinct apex types capable of submolecular imaging. We characterize our tip apices by "inverse imaging" of the silicon adatoms of the $\mathrm{Si}(111)-7 \times 7$ surface and support our findings with detailed density functional theory (DFT) calculations. We also show that intramolecular resolution on individual molecules may be readily obtained at $78 \mathrm{~K}$, rather than solely at $5 \mathrm{~K}$ as previously demonstrated. Our results suggest a wide range of tips may be capable of producing intramolecular contrast for molecules adsorbed on semiconductor surfaces, leading to a much broader applicability for submolecular imaging protocols.
\end{abstract}

DOI: 10.1103/PhysRevB.90.165425

PACS number(s): 82.37.Gk, 68.37.Ps, 71.15.Mb, 81.05.ub

\section{INTRODUCTION}

Since Gross et al. [1] first demonstrated striking submolecular resolution in NC-AFM using CO-functionalized tips on the $\mathrm{Cu}(111)$ surface, the ability to image the detailed substructure of molecules adsorbed on metal surfaces has produced a number of impressive results. In addition to further developments by Gross and co-workers related to the bond order of organic molecules [2], localization of charge within a molecule [3], and the suitability of different types of tip apex termination [4], other groups have used a similar protocol to image the stages of a chemical reaction [5], different conformations of an adsorbed molecule [6], and, very recently, intermolecular contrast in regions where hydrogen bonding is expected [7-9]. This is in addition to the scanning tunneling hydrogen microscopy (STHM) technique pioneered by Tautz and Temirov [10,11], which also demonstrated similar submolecular and intermolecular contrast.

Nonetheless, it is notable that submolecular resolution has only been obtained on bare metal and passivated substrates (such as $\mathrm{Ag} / \mathrm{Si}(111)$ [8] or $2 \mathrm{NaCl} / \mathrm{Cu}(111)$ [1]), usually employing specific STM techniques to controllably functionalize the tip. An important unanswered question is whether this technique is generalizable across different surfaces, including, in particular, highly reactive semiconductor surfaces.

We find that submolecular resolution is routinely achieved when imaging a simple prototypical planar organic molecule [naphthalene tetracarboxylic diimide (NTCDI)] on the

\footnotetext{
*Corresponding author: adam.sweetman@ @ottingham.ac.uk

${ }^{\dagger}$ These authors contributed equally to this work.
}

Published by the American Physical Society under the terms of the Creative Commons Attribution 3.0 License. Further distribution of this work must maintain attribution to the author(s) and the published article's title, journal citation, and DOI.
Si(111) $-7 \times 7$ substrate. Remarkably, we find that two distinct tip apices, each having a very different chemical interaction with the silicon adatoms, produce submolecular resolution rivaling that of CO-terminated tips on metal surfaces, and obtain this resolution without deliberate functionalization of the tip. We utilize "inverse imaging" of a well-known surface structure [12-15], combined with density functional theory (DFT) calculations, to elucidate different tip terminations that may be capable of producing submolecular resolution. We interpret our results in the context of spontaneous functionalization of the tip via different possible apex terminations including NTCDI, $\mathrm{OH}$, or relatively unreactive silicon clusters.

\section{EXPERIMENTAL METHODS}

Data were acquired using an Omicron Nanotechnology LT-STM/NC-AFM operating in UHV at cryogenic temperatures. Clean $\mathrm{Si}(111)-7 \times 7$ surfaces were prepared by flash annealing the silicon wafers to $1200^{\circ} \mathrm{C}$, rapid cooling to $900{ }^{\circ} \mathrm{C}$, and then slow cooling to room temperature. A low coverage of NTCDI was prepared by depositing the molecules from a standard Knudsen cell (heated to approximately $230{ }^{\circ} \mathrm{C}$ ) onto the room temperature substrate. Post-deposition, the sample was transferred into the scan head and left to cool before imaging.

Commercial qPlus sensors (Omicron $\mathrm{GmbH}$ ) with electrochemically etched tungsten wire tips were introduced into the scan head without any further preparation. The sensors were first prepared on clean $\operatorname{Si}(111)-7 \times 7$ surfaces by standard STM techniques until good STM/NC-AFM resolution was achieved. Similar techniques were used to recondition the tip on the surface after molecular deposition, which may facilitate transfer of adsorbed molecules onto the tip apex. For the qPlus sensor used in this study we typically recorded resonant frequencies of $\sim 25 \mathrm{kHz}$, and, based on previous measurements of similar sensors [16], assume an effective stiffness of $k \sim 2000 \mathrm{~N} / \mathrm{m}$. All NC-AFM imaging was performed at 
$0 \mathrm{~V}$ bias in order to eliminate any possible effect from either electronic crosstalk [17] or the so-called "phantom force" [18].

Typically we first image a region of interest in STM mode, and allow any drift or piezoelectric creep to stabilize, before transferring to imaging the same region in constant height NCAFM. During constant height imaging, oscillation amplitudes $\left(A_{0}\right)$ of between 0.1 and $0.3 \mathrm{~nm}$ were used. To transfer from STM to constant height NC-AFM, the tip was withdrawn $\sim 1$ to $5 \mathrm{~nm}$ from the surface and the gap voltage between tip and surface slowly reduced to $0 \mathrm{~V}$. Subsequent measurements were taken out of feedback over the same region and the tip slowly approached to the surface until submolecular resolution was achieved.

An atom tracking system [19] was used to apply feedforward correction when multiple images of the same region were required. In the discussion of our experimental results below, $z$ heights for image sequences are given relative to the tip height at the $\Delta f$ value used for feedback during atom tracking. In order to measure site-specific interaction forces, single point $\Delta f(z)$ spectroscopy measurements were performed on the adatoms, molecules, and the cornerholes. The cornerhole spectrum was then subtracted from the "on" spectra (i.e., the so-called on-minus-off subtraction method [20-22]) and the resultant short-range $\Delta f(z)$ was inverted to force using the Sader-Jarvis algorithm [23]. Therefore, all experimental forces presented in this paper are short range (i.e., site specific), and are directly compared to simulation. All images presented are shown as raw data, with the exception of topographic STM images which have had background slope removed by a plane fit.

In the extraction of the $F(z)$ curves it must be noted that a number of uncertainties are present that may quantitatively affect the quoted force values. First, due to uncertainties in the amplitude calibration procedure we would expect an uncertainty in the quoted oscillation amplitudes of around $10 \%$. Second, a large variation in the effective stiffness of the qPlus sensors used has been observed, most likely due to variance in the gluing between different sensors. Consequently, while we estimate $k$ values of $2000 \mathrm{~N} / \mathrm{m}$, with a likely error of $\sim 10 \%$, outliers have occasionally been observed with variations up to $\sim 30 \%$. We note, however, that as the same sensor was used for all measurements in this study any uncertainty will affect all the results systematically.

\section{SIMULATION METHODS}

We calculated $F(z)$ curves from DFT with the ab initio SIESTA code [24] using a double- $\zeta$ polarized basis set with the GGA Perdew-Burke-Ernzerhof (PBE) density functional and norm-conserving pseudopotentials. Atomic relaxation was considered complete when forces on atoms were smaller than $0.02 \mathrm{eV} / \AA$. The $\mathrm{Si}(111)-7 \times 7$ surface was modeled as a single adatom with a 15-atom silicon cluster terminated with hydrogen atoms [13]. Five atoms (green in Fig. 4) within the "T4" configuration were allowed to relax [13]. To test for the possibility of NTCDI-terminated tips we used a single NTCDI molecule in three orientations with either the C-O, $\mathrm{N}-\mathrm{H}$, or two $\mathrm{C}-\mathrm{H}$ groups pointing down, which we term Odown, H-down, and side-down, respectively. We also explored the behavior of bare and $\mathrm{OH}$-passivated silicon dimer tip
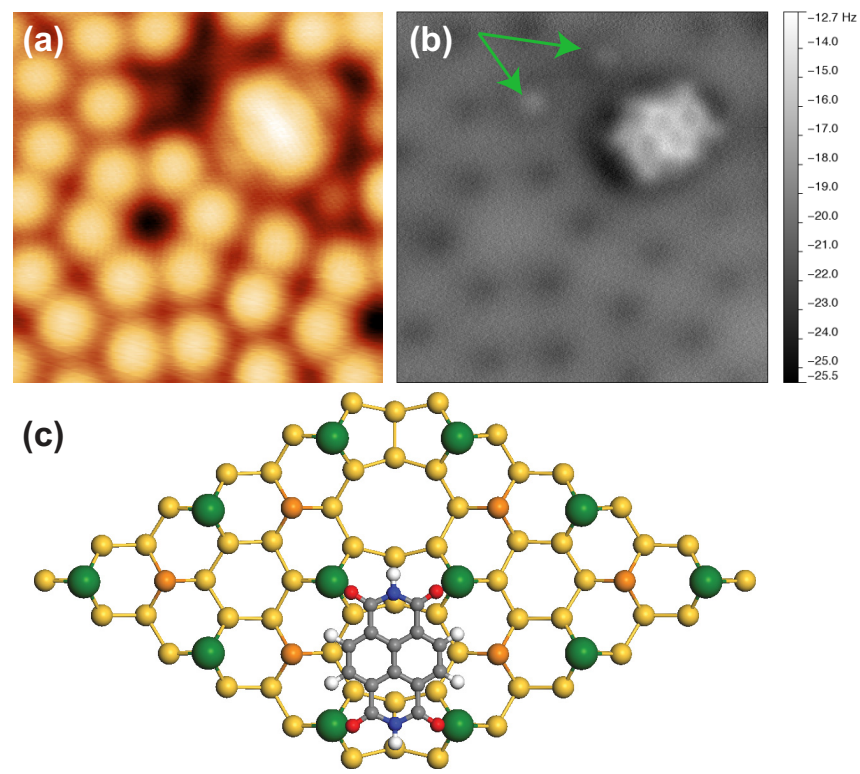

FIG. 1. (Color online) (a) STM image taken of an NTCDI molecule adsorbed on the $\mathrm{Si}(111)-7 \times 7$ surface at $78 \mathrm{~K}$, image parameters $\mathrm{V}_{\text {gap }}=+1.5 \mathrm{~V}, I_{t}=40 \mathrm{pA}$. (b) Constant height NC-AFM $\Delta f$ image, taken with the same tip. Oscillation amplitude $A_{0}=0.28 \mathrm{~nm}$. Image size $3.8 \mathrm{~nm} \times 3.8 \mathrm{~nm}$. Green arrows highlight nearby passivated silicon adatoms. (c) Ball and stick model of NTCDI adsorbed on the $\mathrm{Si}(111)-7 \times 7$ unit cell.

apexes. To calculate $F(z)$ curves the NTCDI and silicon tips were placed at an initial vertical position of $7 \AA$ above the $\mathrm{T} 4$ adatom. The vertical distance $z$ is defined as the distance between the vertical position of the adatom and the lowest tip apex atom prior to relaxation. The tip was then moved in quasistatic steps approaching the surface to just beyond the force turning point, and then retracted in the same way. At each point the vertical forces acting on the fixed tip atoms were summed up to give the total force acting on the tip.

\section{EXPERIMENTAL RESULTS}

\section{A. Intramolecular imaging with NC-AFM}

In Fig. 1 we show representative constant current STM (a) and constant height NC-AFM (b) images of an NTCDI molecule adsorbed on the $\mathrm{Si}(111)-7 \times 7$ surface. Figure 1(a) shows features typical of a molecule imaged with STM. STM is primarily sensitive to the molecule's local density of states within an energy window set by the tip-sample bias, and thus image interpretation can often be challenging. Figure 1(b) shows the same region imaged in constant height NC-AFM mode. The most striking feature in Fig. 1(b) is the clear submolecular resolution obtained within the NTCDI molecule itself, but we also clearly resolve the surface adatoms as attractive depressions, and what appear to be passivated silicon adatoms to the upper left of the NTCDI (see green arrows) [25]. Simultaneous resolution of the molecule and surface makes it possible to identify the bonding configuration of the NTCDI molecule on the surface, as shown in Fig. 1(c). It is also noteworthy that single molecule intramolecular contrast is obtained here using $\mathrm{LN}_{2}$ at $78 \mathrm{~K}$, whereas previous 


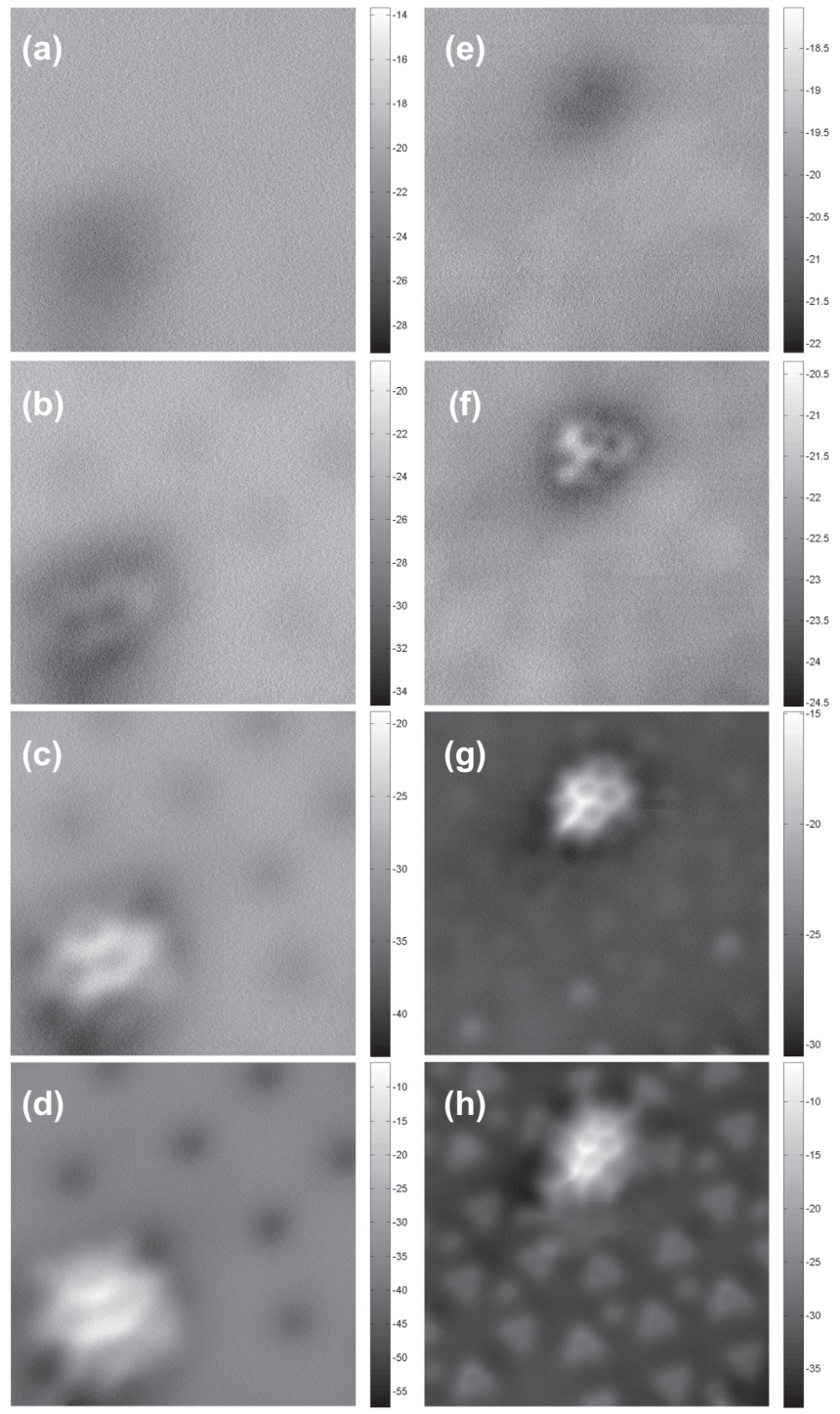

FIG. 2. (a)-(d) Series of constant height images taken at decreasing tip-sample separation with a tip demonstrating attractive adatom contrast. Relative tip heights $0,-0.075,-0.14$, and -0.20 $\mathrm{nm}$, respectively. $A_{0}=0.11 \mathrm{~nm}$. Image size $2.2 \mathrm{~nm} \times 2.2 \mathrm{~nm}$. (e)-(h) Series of constant height images with a tip demonstrating repulsive adatom contrast. Relative tip heights $0,-0.08,-0.19$, and $-0.28 \mathrm{~nm}$, respectively. $A_{0}=0.28 \mathrm{~nm}$. Image size $3.5 \mathrm{~nm} \times 3.5 \mathrm{~nm}$. Datasets acquired at $5 \mathrm{~K}$.

results on metal surfaces have required LHe cooling to $5 \mathrm{~K}$. This is most likely due to the stronger molecule-surface interaction present on semiconductor surfaces, which will suppress diffusion of molecules adsorbed on either the tip or surface.

In Fig. 2 two series of constant height images [(a)-(d) and (e)-(h)] are shown, taken with different tip apices, at decreasing tip-sample separation, showing the progression in contrast as the tip approaches the surface. Since we do not deliberately functionalize our tip apices, it is perhaps somewhat surprising that we observe submolecular contrast over the molecules. Interestingly, similar submolecular contrast on the NTCDI molecules is obtained with two very different contrasts on the adatoms. This variation in adatom imaging suggests that there are at least two characteristic classes of tip apex capable of producing submolecular resolution. For convenience we will refer to tips of the type shown in Figs. 2(a)-2(d) as "attractivetype" tips, and in Figs. 2(e)-2(h) as "repulsive-type" tips for the rest of the paper. We stress that, in common with most SPM experiments where the tip is not controllably terminated, we also observe a range of contrasts that show unclear or distorted imaging that is not so easily interpreted. Nonetheless, for tips that produced good STM contrast we regularly observe the intramolecular contrast presented above, suggesting that we regularly form tip structures with the properties required for submolecular imaging.

For attractive-type tips we see contrast similar to that shown in Fig. 1. Far from the surface we see the attractive potential well associated with the molecule, and on closer approach submolecular features are resolved. Critically, the contrast on the adatoms does not change significantly, becoming more attractive as the tip approaches the surface. Conversely for repulsive-type tips, while we observe a very similar progression in contrast on the molecule, the evolution of the contrast on the adatoms is radically different. Far from the surface we observe a very weak attractive interaction, but on closer approach the interaction becomes repulsive, resulting in a strikingly clear repulsive map of the surface, resolving both adatoms and rest atoms. (We also note the clear triangular shape of the adatoms in this image, which we address elsewhere [26]).

In the sequence shown in Figs. 2(e)-2(h), the molecule is adsorbed in a cornerhole, and hence is in a lower position relative to the adatoms than the molecules in Fig. 1 or Figs. 2(a)-2(d). This might at first be thought to be the origin of the difference in the evolution of contrast above the adatoms. However, this particular configuration of the molecule was chosen specifically to allow continuous imaging of both it and the adatoms simultaneously at close approach. When imaging molecules adsorbed on top of the adatoms with this tip type, it is generally necessary to withdraw the tip some distance $(\sim 100$ to $150 \mathrm{pm}$ ) from the height at which repulsive imaging of the adatoms is carried out to allow stable images of the molecule to be acquired [27,28]. This is demonstrated in Fig. 3, which shows images of NTCDI molecules adsorbed on top of the $\mathrm{Si}(111)-7 \times 7$ adatoms, acquired with tips demonstrating repulsive adatom contrast. At the position marked by the arrows the tip was withdrawn from the surface by the distance noted in each figure to allow stable imaging over the molecules. At the molecule imaging height weak attractive imaging of the adatoms can sometimes be observed. Additionally, from the force data obtained during each set of experiments (see below), we observe a qualitatively different interaction over the adatoms with the different tip types. Consequently, the progression in contrast observed in the sequence above cannot be ascribed to the cornerhole adsorption geometry.

\section{B. Force spectra and tip characterization}

Before turning to discussion of the nature of the tip structure, it is instructive to consider some general principles underpinning submolecular resolution imaging in NC-AFM. In light of recent work on metal surfaces investigating the performance of different tip terminations capable of 

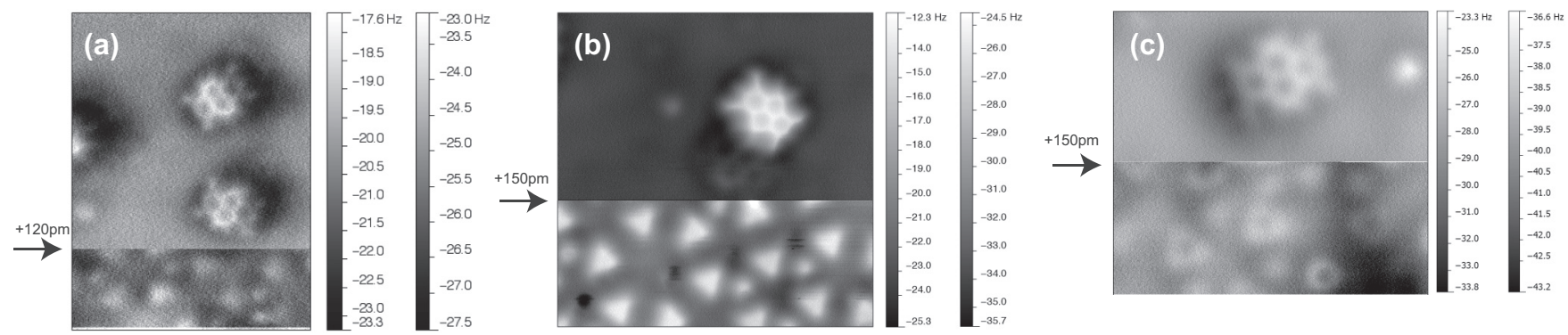

FIG. 3. Additional images of NTCDI acquired with tip apices which yield repulsive adatom contrast at (a) $78 \mathrm{~K}$ and (b) and (c) $5 \mathrm{~K}$. The left-hand color bars give the $\Delta f$ scales for the upper parts of each images (molecules), the right-hand bars for the lower part (adatoms). Note the tip in image (b) shows a slight double feature over the molecule suggesting either multiple, or tilted, molecular adsorption on the tip.

intramolecular contrast [4,29], it seems that a general scheme can be suggested. In order for intramolecular contrast to be obtained three primary conditions must be met: (1) The tip must not react strongly with the target molecule; (2) the tip must be atomically sharp; and (3) the total force between tip and molecule at the imaging height necessary to access the repulsive force regime must not exceed the bonding force between the tip and surface (or, trivially, the force needed to destabilize the tip apex). In particular, we will return to discussion of condition (3) later in the paper.

Figure 4(a) shows experimental short-range $F(z)$ measurements acquired over the NTCDI molecule and adatoms with both types of tip apex. In the dataset taken with attractive adatom contrast, we see a smooth turnaround on the molecule with a peak force of $\sim 150$ to $200 \mathrm{pN}$. On the adatoms we see a strong attractive force, with a peak attraction of $\sim 2 \mathrm{nN}$. In the data acquired with the repulsive adatom contrast, we see a similar interaction over the molecule (peak force $\sim 100$ to $150 \mathrm{pN})$, but an extremely weak interaction with the adatoms ( $\sim 10$ to $50 \mathrm{pN})$, strongly suggesting a passivated tip [30-32].

Consequently, we are able to determine that there are two very different tip terminations capable of producing submolecular resolution, with similar interactions with the adsorbed molecules, but with significantly different interactions with the adatoms of the silicon surface.

\section{Simulation results}

In order to elucidate the nature of the tip apex, we modeled the interaction of a number of tip apices with a surface silicon adatom. Experimentally it is clear that neither tip reacted strongly with the surface-adsorbed NTCDI, while we observe two radically different interactions with the adatoms. In Fig. 4 we compare the experimental $F(z)$ curves with those calculated from DFT simulated force spectra for a number of plausible tip models.

These models were selected because, as a consequence of our tip preparation techniques, we not only expect our tips to be silicon-terminated, but also that both the surfaceadsorbed NTCDI molecules, and any other common surface containments (such as dissociated $\mathrm{OH}$ or $\mathrm{H}$ ), can be readily transferred to the tip apex.

\section{Repulsive-type tips}

We first consider the tip models which seem the most plausible candidates to produce the repulsive-type contrast on the adatoms observed experimentally. As shown in Fig. 4(b), for the hydrogen-down NTCDI tip and $\mathrm{OH}$-terminated silicon tip we observe almost no attractive interaction. Instead, we observe that the forces become positive as the tips approach towards the adatom. This is consistent with previous work
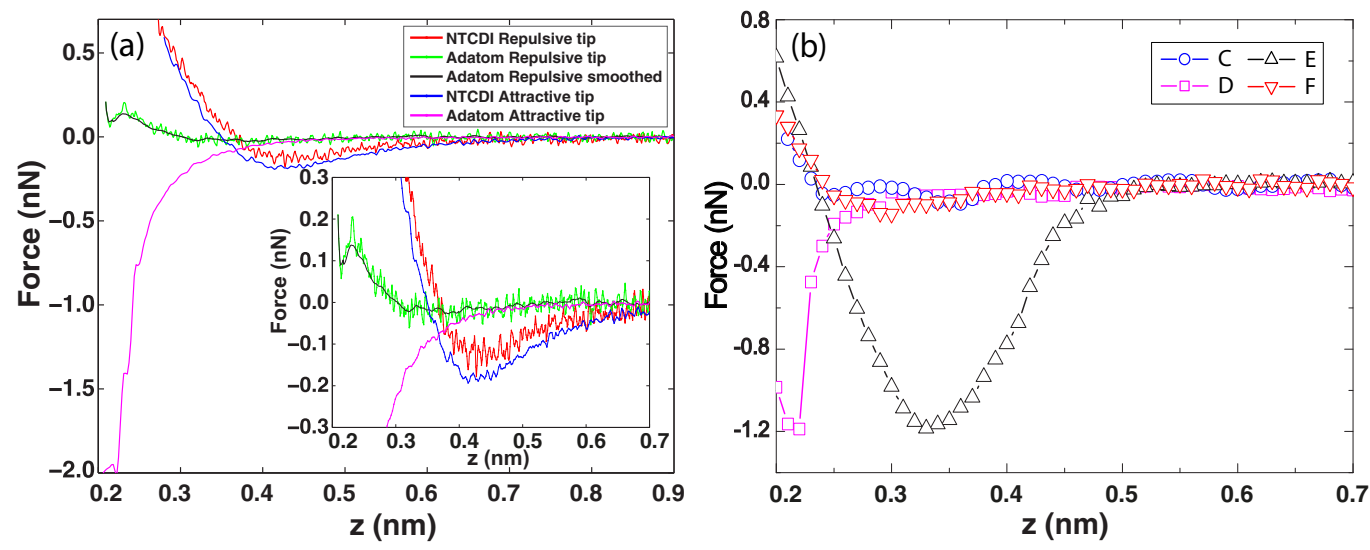

(c)

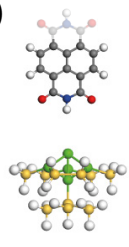

(d)

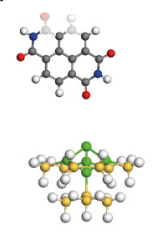

(e)

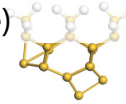

(f)
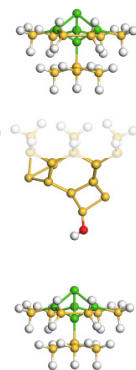

FIG. 4. (Color online) (a) Experimental tip-sample forces $F(z)$ on an NTCDI molecule and a $\operatorname{Si}(111)-7 \times 7$ adatom for attractive and repulsive adatom contrasts (data taken from the sequences shown in Fig. 2). Note that the attractive adatom data has been shifted +80 pm on the $z$ axis to align the minima of the molecular interaction curves of the two datasets. Inset: Experimental data from (a) showing detail of the molecular force turnaround. (b) Calculated $F(z)$ curves modeled using DFT for the four tip models shown in (c)-(e), over a silicon adatom. Fixed tip atoms are grayed out while surface atoms which are free to relax are shaded in green. 

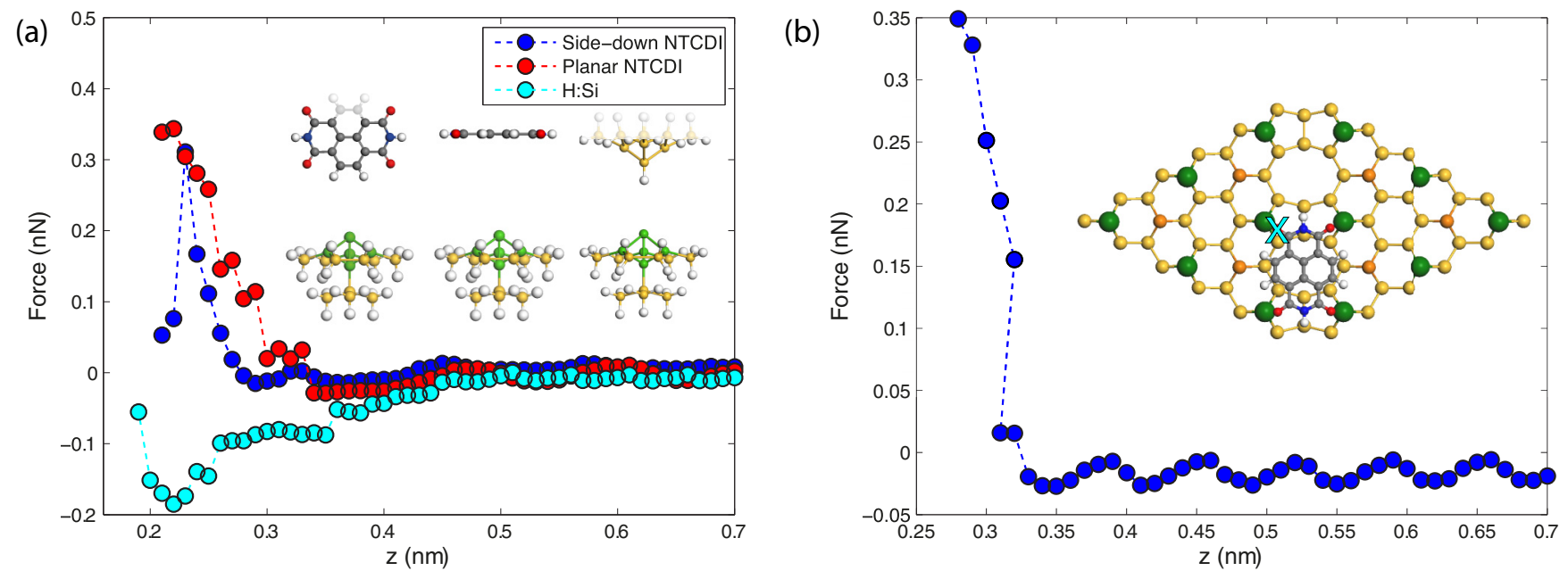

FIG. 5. (Color online) (a) Simulated $F(z)$ for three additional tip terminations: two additional NTCDI tips (side down and planar) and an additional H-terminated H3-type silicon cluster (tip models shown inset). (b) Simulated $F(z)$ for a bare silicon dimer tip over the oxygen atom of a surface-adsorbed NTCDI molecule (location marked with an " $x$ " in the inset diagram).

which observed that $\mathrm{H}$ or $\mathrm{OH}$ tip terminations suppress the chemical attraction [30,32]. Both of these tips are passivated, and, by analogy to the imaging mechanism described for CO-terminated metal tips [1], are likely to be able to produce submolecular contrast while also resulting in repulsive contrast on the silicon adatoms.

On the basis of our simulations alone we cannot clearly distinguish between these two tip structures, but a recent theoretical study [33] suggests that electropositive tips such as the H-down NTCDI apex could react strongly with the $\mathrm{Si}(111)-7 \times 7$ rest atoms. On the basis of the repulsive contrast over both adatoms and rest atoms observed experimentally, and noting the observation of similar contrast on $\operatorname{Si}(111)-7 \times 7$ for tips prepared in the absence of adsorbed NTCDI molecules [26], we tentatively suggest the $\mathrm{OH}-$ terminated tip model may be a more plausible candidate.

For completeness we also show in Fig. 5(a) alternative DFT results calculated for additional repulsive-type tip clusters, specifically a hydrogen passivated silicon cluster (H3 type) and tip adsorbed NTCDI molecule with the NTCDI either side-on (i.e., the two $\mathrm{C}-\mathrm{H}$ bonds on the long edge) or planar (i.e., the carbon backbond planar to the surface normal) with respect to the silicon adatom. While the NTCDI spectra here are almost identical to the H-down spectra described above, we note that these orientations of the NTCDI do not present a single atom towards the surface, and so we would not expect them to be capable of producing submolecular contrast similar to that shown in the images above.

\section{Attractive-type tips}

We now turn to the tip models which are possible candidates for the attractive-type contrast on the silicon adatoms. For the O-down NTCDI orientation and bare silicon tip we observe a strong attractive interaction with the surface silicon adatom, originating from covalent bond formation between the silicon and terminating tip atom. We note that while the silicon tip experiences a relatively smooth increase in force, the
O-down NTCDI tip experiences a rapid jump to contact due to deformation of the tip-NTCDI, causing the oxygen lone pair to more favorably align with the silicon adatom. On the basis of the more pronounced relaxation of the tip, sharper "jump-to-contact," and recent work suggesting that an O-down NTCDI tip should allow for imaging comparable to that of the $\mathrm{CO}$ terminated tips used in previous studies on metal surfaces [8], we suggest that the O-down NTCDI tip is a more likely candidate for the experimental dataset presented.

Nonetheless, there is reason to suspect that a siliconterminated cluster could also produce similar results. It might intuitively be expected that a silicon-terminated cluster would react strongly with the NTCDI molecule, but recent theoretical work [34] suggests that some dimerlike tips may react relatively weakly with closed carbon systems. As shown in Fig. 5(b), our own calculations suggest this is also true in the case of the surface-adsorbed NTCDI. From our results it is clear that the oxygen atom is the most reactive part of the NTCDI molecule. Consequently, the oxygen site will have the highest probability to strongly interact with a silicon tip. Therefore, in Fig. 5(b) we show $F(z)$ simulated for a bare silicon dimer tip positioned over the oxygen atom of a surface-adsorbed NTCDI. Surprisingly, we observe no discernible chemical attraction between the tip and surfaceadsorbed molecule, most likely due to the pre-existing bonding between the molecule and surface adatoms.

Consequently, it seems possible that the silicon-terminated dimer tip should also be able to approach into the repulsive regime over the whole molecule and potentially produce submolecular contrast without destabilizing the molecule. Returning to the three conditions for submolecular imaging described above, we note that we do not expect that stable close approach is possible for tips of this type where the molecule does not bond strongly to the surface (e.g., on metals and passivated substrates), nor that the same argument will hold for more reactive silicon clusters capable of causing rehybridization over the molecule itself [34]. In particular, the saturation of reactive molecular moieties (i.e., the four 
oxygen groups of the NTCDI in this instance) inhibits bond formation with the tip and may consequently permit repulsive mode imaging with a wider range of tips than might otherwise be possible. As such, it is possible that in certain conditions imaging on on semiconductor surfaces may offer certain advantages with respect to condition (3).

Although we make a qualitative assignment of the likely tip terminations present in our experimental results, we observe quantitative differences between the experimental and calculated forces for the attractive imaging tips. The DFT calculations predict a lower peak force for both tips than is measured experimentally $(\sim 1.2 \mathrm{nN}$ compared to $\sim 2 \mathrm{nN})$. We first note that our DFT calculations do not include contributions due to local dispersion (i.e., short range van der Waals) interactions, and as such we expect them to systematically underestimate the experimentally observed forces. We also note that our simulations appear to systematically underestimate the tip-sample forces for well-studied silicon clusters compared to similar simulations performed using a different DFT code [32]. Finally we emphasise it is likely that our simplified tip models do not fully capture the complex tip structure present experimentally. Consequently, we are careful here to only use the DFT calculations as a qualitative guide to the behavior of certain tip "classes," and not to assign definitive tip structures based solely on comparison to the absolute magnitude of the calculated forces.

Nonetheless, our calculations strongly suggest that a broad range of tip types may be capable of producing submolecular contrast in NC-AFM experiments on semiconducting surfaces. In particular, the strong bonding of the molecules to the surface may allow certain tip structures to enter the repulsive regime over the molecules even without the use of the passivation strategy required for metal substrates.

\section{Detailed DFT simulation of NTCDI tips on Si adatom}

Although the interaction properties of the siliconterminated clusters discussed above have been well investigated theoretically, the interaction of the NTCDI molecules with the adatom cluster is not yet well characterized. In order to explore this system more fully, simulated force images of a tip-adsorbed NTCDI molecule interacting with a silicon adatom of the $\mathrm{Si}(111)-7 \times 7$ surface were constructed by calculating the tip-sample force in three dimensions (3D). The 3D force field was determined by performing multiple $F(z)$ simulations across a predefined $x-y$ grid [35]. Two tip orientations were chosen, $\mathrm{H}$-down and O-down, representing the two terminations of the molecule most likely to provide submolecular resolution. The symmetry of the silicon adatom allows the number of calculations to be reduced significantly: for both cases a $3 \AA$ by $6 \AA$ irreducible region with a lateral spacing of $0.375 \AA$ (or $9 \times 18$ points) and a vertical spacing of $0.1 \AA$ was chosen. The full image is constructed from a total of 289 points by breeding the irreducible region.

Figures 6(a) and 6(b) show calculated constant height force slices for the O-down and H-down tips, respectively, at what is considered to be a typical tip-sample separation during image acquisition. For the H-down tip we observe similar bright contrast to the experimental images due to strong repulsion between the tip hydrogen and surface adatom
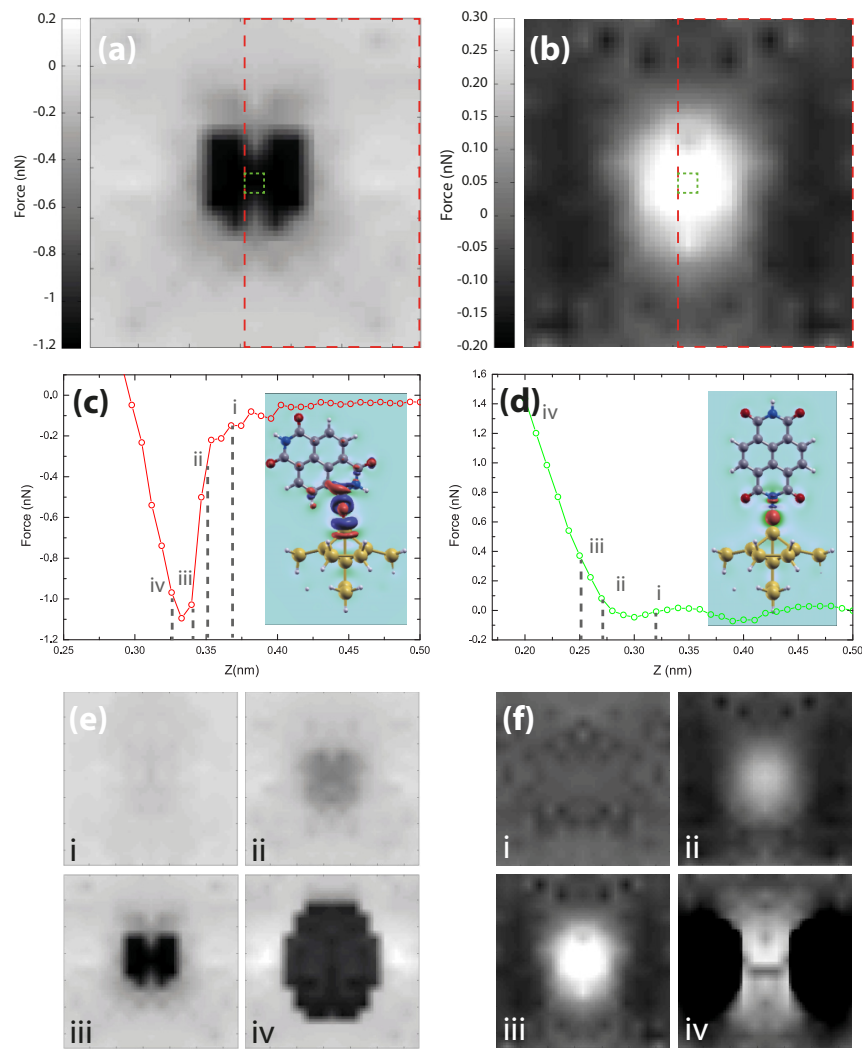

FIG. 6. (Color online) Simulated constant height force slices for an NTCDI terminated tip on a $\mathrm{Si}(111)-7 \times 7$ adatom. (a) Calculated constant height force slice with the O-down tip shows the adatom as a bright repulsive feature. The red dashed box marks the calculated points which are then bred onto the rest of the image. The green dotted box shows the uninterpolated pixel size, the $F(z)$ at this point is shown in (c) with an inset electron density different plot illustrating the electron repulsion between the tip and sample at an isovalue of $0.02 e /$ bohr $^{3}$. (e) Progression of constant height slices at decreasing tip-sample separations at heights marked by i-iv in (c). (b), (d), and (f) Equivalent data for an $\mathrm{H}$-down tip orientation.

[see Fig. 6(d)]. One noticeable difference, however, is the absence of the clearly resolved triangular features. This can perhaps be attributed to the simplifications made with respect to the symmetry of the tip, and the T4 cluster approximation, which is less justified far into the repulsive part of the tip-sample interaction.

The full range of interaction considered in this calculation is shown in Fig. 6(f) as a progression of constant height force slices at decreasing tip-sample separations. We stress that local $\mathrm{vdW}$ interactions are not considered in our calculations. Thus the simulated images show the bright repulsive feature without any preceding weak attraction. As the tip-sample distance is decreased the repulsive feature becomes clearer until the side oxygen groups also start to interact with the silicon adatom. At these distances the forces experienced by the molecular tip exceed the range typically explored in experiment.

In contrast, for the O-down tip we observe attractive behavior characterized by the silicon adatom appearing as a dark depression. In Fig. 6(a) at a particular tip-sample separation a double lobe feature can be observed corresponding to the lone 


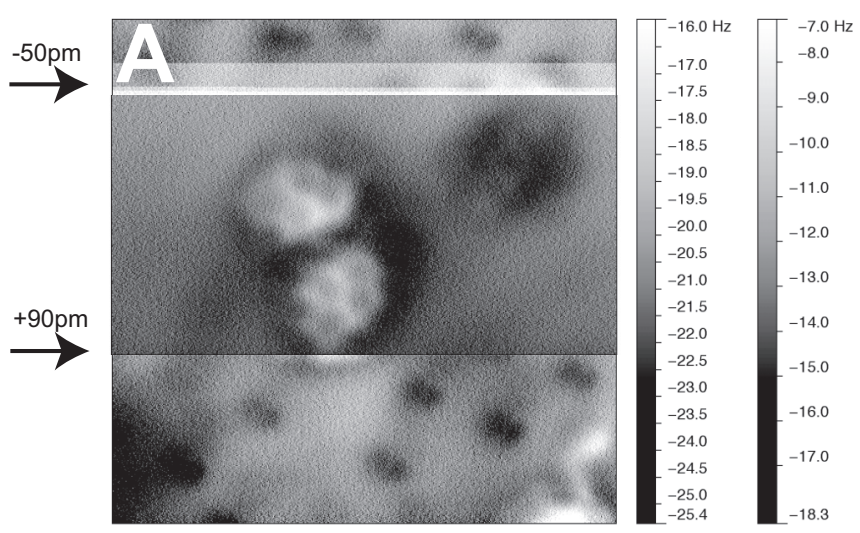

FIG. 7. Image acquired of two NTCDI molecules with a "double lobe" attractive tip. Arrows indicate positions where the tip height was altered relative to the starting tip height (slow scan direction bottom to top). Right-hand color bar corresponds to contrast over the molecules, left-hand bar to contrast over the adatoms. $A_{0}=0.275 \mathrm{~nm}$. Image acquired at $78 \mathrm{~K}$.

pairs of the oxygen atom. In our simulations the tip-NTCDI molecule deforms to allow favorable alignment of the lone pair with the silicon adatom. In Fig. 6(e) a progression of constant height force slices and an electron density difference plot [Fig. 6(c) inset] is shown illustrating the formation of the covalent bond responsible for the attractive features. While our calculations suggest that a double lobe feature should be initially observed, the range in $z$ where each lobe is clearly identifiable is only of the order $30 \mathrm{pm}$. Beyond this point our calculations show that the predominant feature would be a single lobe, similar to the majority of experimental observations.

However, although we do rarely observe double lobe contrast experimentally (Fig. 7) it should be noted that caution should be applied in conclusively assigning its origin to subatomic imaging of the lone pairs on the oxygen atom of a tip adsorbed NTCDI. The evolution in imaging shown theoretically may not occur for a more rigid attachment of the molecule to the tip, nor for a misaligned molecule, as either situation could prevent the required alignment of the lone pair to the silicon adatom. It is also important to note that similar double lobe features can in principle arise from contaminated silicon dimer tips [36], and that such tips may, in principle, also be capable of producing intramolecular contrast as described above for the case of clean dimer tips.

\section{CONCLUSIONS}

We have presented a submolecular resolution noncontact force microscopy imaging of planar molecules on a reactive semiconducting substrate. Submolecular contrast is routinely observed, despite the lack of deliberate tip functionalization. Intriguingly, we identify two distinct classes of tip apex capable of producing submolecular resolution, based on their relative interaction strengths with the NTCDI molecule and the surrounding silicon adatoms. By comparing our experimental results with simulated force spectra, we assign these different types of contrast to either different orientations of an NTCDI molecule adsorbed on the tip or a mix of passivated and bare silicon tip apices. This observation of "bimodal" submolecular contrast on a semiconductor, without deliberate tip functionalization (and at $\mathrm{LN}_{2}$ temperatures), suggests that this technique may have a much wider applicability than has thus far been demonstrated, and opens the way for similar measurements on a raft of other molecule-surface systems.

\section{ACKNOWLEDGMENTS}

P.M., S.P.J., and A.S. thank the Engineering and Physical Sciences Research Council (EPSRC) and the Leverhulme Trust, respectively, for Grants No. EP/G007837/1, No. EP/J500483/1, and No. F00/114 BI. We are grateful for access to the University of Nottingham High Performance Computing Facility. N.R.C. gratefully acknowledges receipt of a Royal Society Wolfson Merit Award. P.R. gratefully acknowledges financial support from the Alexander von Humboldt-foundation.
[1] L. Gross, F. Mohn, N. Moll, P. Liljeroth, and G. Meyer, Science 325, 1110 (2009).

[2] L. Gross, F. Mohn, N. Moll, B. Schuler, A. Criado, E. Guitian, D. Pena, A. Gourdon, and G. Meyer, Science 337, 1326 (2012).

[3] F. Mohn, L. Gross, N. Moll, and G. Meyer, Nat. Nanotechnol. 7, 227 (2012).

[4] F. Mohn, B. Schuler, L. Gross, and G. Meyer, Appl. Phys. Lett. 102, 073109 (2013)

[5] D. G. de Oteyza, P. Gorman, Y. C. Chen, S. Wickenburg, A. Riss, D. J. Mowbray, G. Etkin, Z. Pedramrazi, H. Z. Tsai, A. Rubio, M. F. Crommie, and F. R. Fischer, Science 340, 1434 (2013).

[6] N. Pavliček, B. Fleury, M. Neu, J. Niedenführ, C. HerranzLancho, M. Ruben, and J. Repp, Phys. Rev. Lett. 108, 086101 (2012).
[7] J. Zhang, P. Chen, B. Yuan, W. Ji, Z. Cheng, and X. Qiu, Science 342, 611 (2013).

[8] A. M. Sweetman, S. P. Jarvis, H. Sang, I. Lekkas, P. Rahe, Y. Wang, J. Wang, N. R. Champness, L. Kantorovich, and P. Moriarty, Nat. Commun. 5, 3931 (2014).

[9] P. Hapala, G. Kichin, C. Wagner, F. S. Tautz, R. Temirov, and P. Jelínek, Phys. Rev. B 90, 085421 (2014).

[10] R. Temirov, S. Soubatch, O. Neucheva, A. C. Lassise, and F. S. Tautz, New J. Phys. 10, 053012 (2008).

[11] C. Weiss, C. Wagner, R. Temirov, and F. S. Tautz, J. Am. Chem. Soc. 132, 11864 (2010).

[12] M. Herz, F. J. Giessibl, and J. Mannhart, Phys. Rev. B 68, 045301 (2003).

[13] C. Chiutu, A. M. Sweetman, A. J. Lakin, A. Stannard, S. Jarvis, L. Kantorovich, J. L. Dunn, and P. Moriarty, Phys. Rev. Lett. 108, 268302 (2012). 
[14] J. Welker, A. J. Weymouth, and F. J. Giessibl, ACS Nano 7, 7377 (2013).

[15] G. Schull, T. Frederiksen, A. Arnau, D. Sánchez-Portal, and R. Berndt, Nat. Chem. 6, 23 (2010).

[16] A. Sweetman, S. Jarvis, R. Danza, J. Bamidele, S. Gangopadhyay, G. A. Shaw, L. Kantorovich, and P. Moriarty, Phys. Rev. Lett. 106, 136101 (2011).

[17] Z. Majzik, M. Setvín, A. Bettac, A. Feltz, V. Cháb, and P. Jelínek, Beil. J. Nanotechnol. 3, 249 (2012).

[18] A. Weymouth, T. Wutscher, J. Welker, T. Hofmann, and F. J. Giessibl, Phys. Rev. Lett. 106, 226801 (2011).

[19] P. Rahe, J. Schutte, W. Schniederberend, M. Reichling, M. Abe, Y. Sugimoto, and A. Kuhnle, Rev. Sci. Instrum. 82, 063704 (2011).

[20] M. A. Lantz, H. J. Hug, R. Hoffmann, P. J. A. van Schendel, P. Kappenberger, S. Martin, A. Baratoff, and H.-J. Gntherodt, Science 291, 2580 (2001).

[21] M. Ternes, C. González, C. P. Lutz, P. Hapala, F. J. Giessibl, P. Jelínek, and A. J. Heinrich, Phys. Rev. Lett. 106, 016802 (2011).

[22] A. Sweetman and A. Stannard, Beil. J. Nanotechnol. 5, 386 (2014).

[23] J. E. Sader and S. P. Jarvis, Appl. Phys. Lett. 84, 1801 (2004).

[24] J. M. Soler, E. Artacho, J. D. Gale, A. García, J. Junquera, P. Ordejón, and D. Sánchez-Portal, Nanotechnology 14, 2745 (2002).
[25] Z. Majzik, B. Drevniok, W. Kamiński, M. Ondráček, A. B. McLean, and P. Jelínek, ACS Nano 7, 2686 (2013).

[26] A. Sweetman, P. Rahe, and P. Moriarty, Nano Lett. 14, 2265 (2014).

[27] G. H. Simon, M. Heyde, and H.-J. Freund, J. Phys.: Condens. Matter 24, 084007 (2012).

[28] F. Mohn, L. Gross, and G. Meyer, Appl. Phys. Lett. 99, 53106 (2011).

[29] G. Kichin, C. Wagner, F. S. Tautz, and R. Temirov, Phys. Rev. B 87, 081408(R) (2013).

[30] P. Sharp, S. Jarvis, R. Woolley, A. Sweetman, L. Kantorovich, C. Pakes, and P. Moriarty, Appl. Phys. Lett. 100, 233120 (2012).

[31] S. Jarvis, A. Sweetman, J. Bamidele, L. Kantorovich, and P. Moriarty, Phys. Rev. B 85, 235305 (2012).

[32] A. Yurtsever, Y. Sugimoto, H. Tanaka, M. Abe, S. Morita, M. Ondráček, P. Pou, R. Pérez, and P. Jelínek, Phys. Rev. B 87, 155403 (2013).

[33] H. Sang, S. Jarvis, Z. Zhou, P. Sharp, P. Moriarty, J. Wang, Y. Wang, and L. Kantorovich [Sci. Rep. (to be published)].

[34] M. Ondráček, P. Pou, V. Rozsíval, C. González, P. Jelínek, and R. Pérez, Phys. Rev. Lett. 106, 176101 (2011).

[35] C. Hobbs and L. Kantorovich, Surf. Sci. Rep. 600, 551 (2006).

[36] A. Campbellová, M. Ondráček, P. Pou, R. Pérez, P. Klapetek, and P. Jelínek, Nanotechnology 22, 295710 (2011). 